\title{
Slow cord dorsum potentials elicited by descending volleys in man
}

\author{
H. S H I M I Z U, K . S H I M O J , Y. M A R U Y A M A, Y. SA TO, \\ H. H A R AY A M A, A N D T. TS U B A K I \\ From the Department of Anaesthesiology, Niigata University School of Medicine, and Department \\ of Neurology, Brain Research Institute, Niigata University, Asahimachi, Niigata, Japan
}

SUM MARY Human cord dorsum potential (CDP) was recorded from the posterior epidural space at the levels of cervical and lumbar enlargements, using epidural catheters as the recording electrodes, in 15 normal volunteers and six patients suffering from amyotrophic lateral sclerosis. In normal subjects the CDP elicited by descending volleys consisted of spike potentials followed by sharp negative and slow positive waves which were very similar to the P1, N1, and P2 potentials, respectively, of the CDP evoked segmentally. By contrast, in all patients with amyotrophic lateral sclerosis both the sharp negative and slow positive waves were absent in the CDP elicited by descending volleys, while the pattern of each component of the segmentally evoked CDP remained unchanged. Thus, the sharp negative and slow positive waves of human CDP elicited by descending volleys might reflect the activity of interneurones and primary afferent depolarisation respectively, produced by impulses through the corticospinal tract.

It is well known that the cord dorsum potential (CDP) evoked by stimulation of dorsal rootlets or peripheral nerves can be recorded from the cord surface in spinal animals (Gasser and Graham, 1933; Eccles, 1964; Schmidt, 1971). Human CDP has also been recorded intrathecally with needle electrodes (Magladery, 1951; Erterkin, 1976). This method, however, involves many risks, and has not been applied for routine clinical testing. Although small and short latency potentials recorded over the spine have been interpreted to be of spinal origin (Cracco, 1973; Matthews et al., 1974; Jones, 1977), several excitable tissues underneath an electrode impede analysis of their origin. In addition, the slow potentials sometimes have proved difficult to record with a surface electrode (Shimoji et al., 1978). A simple, safe method of recording human CDP has been developed using an epidural catheter as a recording electrode (Shimoji et al., 1971), and its potential pattern has been shown to resemble that of CDP directly recorded from the cord surface in animals (Shimoji et al., 1976, 1977). Here we report slow negative-positive potentials recorded from the posterior epidural space at the

Address for correspondence and reprint requests: Dr H. Shimizu, Department of Anesthesiology, Niigata University School of Medicine, Asahimachi, Niigata 951, Japan. lumbar enlargement, elicited by volleys descending through the cord in normal man, while these potentials are absent in patients suffering from amyotrophic lateral sclerosis. The finding suggests that these slow negative-positive potentials might correspond to interneuronal activities and primary afferent depolarisation (PAD) respectively, produced by descending volleys in the corticospinal tract.

\section{Method}

Fifteen normal volunteers (11 men, four women, aged 20-52 years) and six patients suffering from amyotrophic lateral sclerosis (four men, two women, aged 42-69 years) participated in this study, having given informed consent. The CDP was recorded from the posterior epidural space at the levels of cervical and lumbar enlargements, using epidural catheters as both the recording and stimulating electrodes (Fig. 1). The recording methods resembled those used in previous in vestigations (Shimoji et al., 1971, 1977) on human CDP with in addition a weak epidural stimulation of the spinal cord. The subjects were placed in the lateral decubitus for fixation of the electrodes. The epidural catheters were first inserted into the 

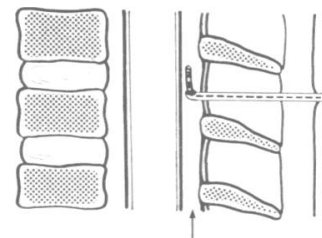

Epidural space

\section{Tuohy needle}

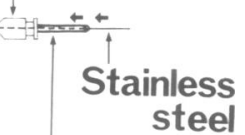

Polyethylene tube

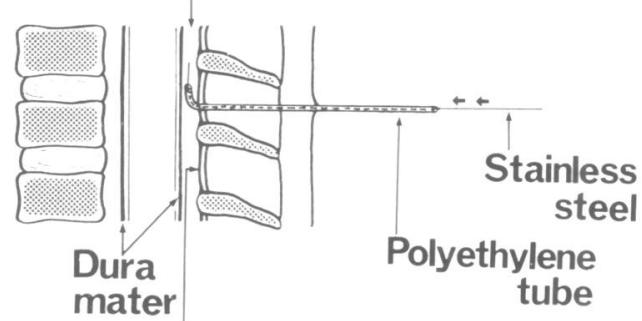

Ligamentum

flavum

Fig. 1 Schematic representation of introduction of electrode into the posterior epidural space of the spine (Shimoji et al., 1971).

posterior epidural space, and then stainless steel wires $(230 \mu \mathrm{m}$ in diameter) were slid inside the catheters up to $5 \mathrm{~mm}$ beyond the tip of the tube (Fig. 1). The reference electrodes (needles, $230 \mu \mathrm{m}$ in diameter) were placed nearby in the supraspinous ligament of the same spinal level. The peripheral stimulating electrodes, paired nonpolarisable silver needles, were inserted into the popliteal fossa or wrist of both sides as close as possible to the peripheral nerves. Stimulus pulses of $0.5 \mathrm{~ms}$ duration were generated by a constant current stimulator with an isolation unit for both the peripheral and epidural stimulations. The evoked response was amplified with a biophysical amplifier having frequency response more than $3 \mathrm{kHz}$ and led to an averaging computer. The time constant used was $0.3 \mathrm{~s}$ for all responses. The background potential changes were also monitored on the pen-writing system. The evoked responses were so small and easily distorted by the electrocardiographic (ECG) artefacts that signal averaging was made at isoelectric intervals between $T$ and $P$ waves of the ECG. The sampling rate was 5-50 points/ms and $10-50$ responses were summed. At the end of each experiment the position of the electrodes in the epidural space was confirmed by radiography.

\section{Results}

The CDP elicited by segmental nerve stimulation consisted of the initially positive spikes (P1) and subsequent slow and sharp negative wave (N1), followed by a slower positive wave (P2) which sometimes had two peaks (Fig. 2a). Several characteristics of these potentials are similar to those of spikes, negative ( $N$ wave) and positive ( $P$ wave) potentials of the CDP in spinal animals (Shimoji et al., 1976, 1977). Therefore, P1, N1, and P2 waves probably reflect the incoming volleys along the roots, interneuronal activities and primary afferent depolarisation respectively. The difference between intact human CDP and CDP in spinal animals has been shown to be the appearance of the second peak in the P2 wave (Shimoji et al., 1976, 1977).

Weak epidural stimulation of the lumbar enlargement, which caused a slight segmental muscle twitch but was not unpleasant, produced polyphasic spikes in the cervical epidural space. On the other hand, weak epidural stimulation of cervical cord produced not only spikes but slow negative-positive complexes in the posterior epidural space of the lumbar enlargement in all normal subjects. These "suprasegmentally evoked" slow potentials were very similar in configuration to those produced segmentally (Fig. 2a). The central latent periods to the peaks of the negative wave and the first component of the slow positive wave in the CDP produced by descending volleys (calculated from the first positive peak of the spike) were approximately $1 \mathrm{~ms}$ shorter than those of the N1 and P2 waves evoked segmentally (Table). These slow potentials evoked by descending volleys, however, could not be demonstrated in any of the six patients who all showed typical signs of amyotrophic lateral sclerosis (muscle weakness, muscular atrophy, fasciculation, hyperreflexia) (Fig. 2b). There were, however, no significant changes in conduction velocities of ascending and descending impulses along the cord in these patients (Table).

\section{Discussion}

The polyphasic spikes recorded from the posterior epidural space elicited by both ascending and descending volleys probably represent the orthodromic or antidromic impulses or both travelling through several pathways in the spinal cord. The subsequent slow negative and positive potentials produced by descending volleys might represent the synchronised potential of interneurones and PAD respectively (Andersen et al., 1964; Fetz, 1968; Schmidt, 1971). In fact, there is evidence that volleys in the corticospinal tract produce PAD in the cat (Andersen et al., 1964; Fetz, 1968; 


\section{PERIPHERAL NERVE STIMULATION}

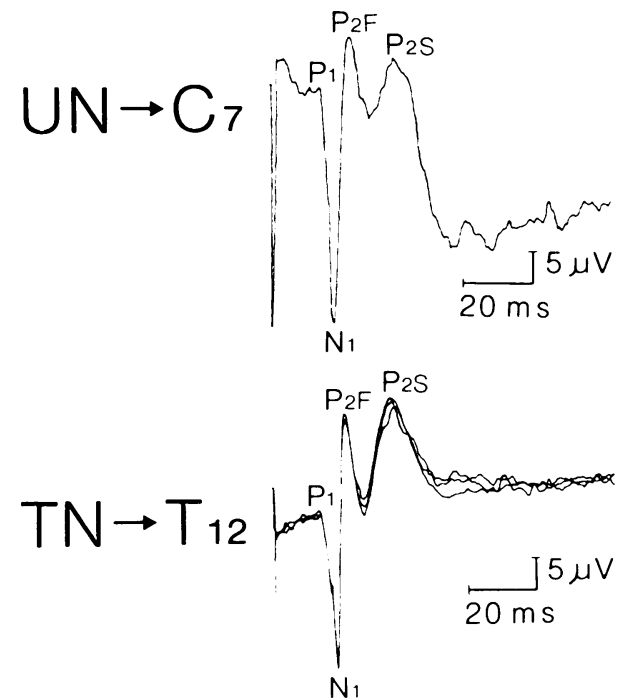

(a)
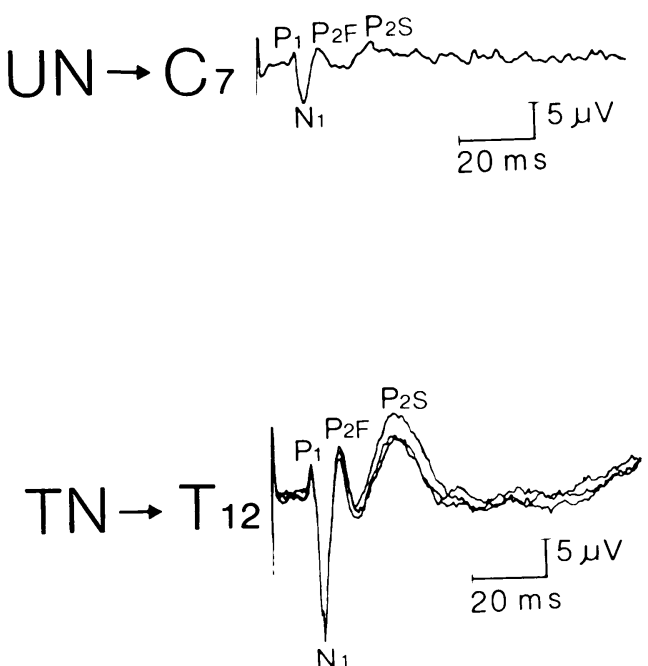

(b)

\section{DORSAL COLUMN STIMULATION}
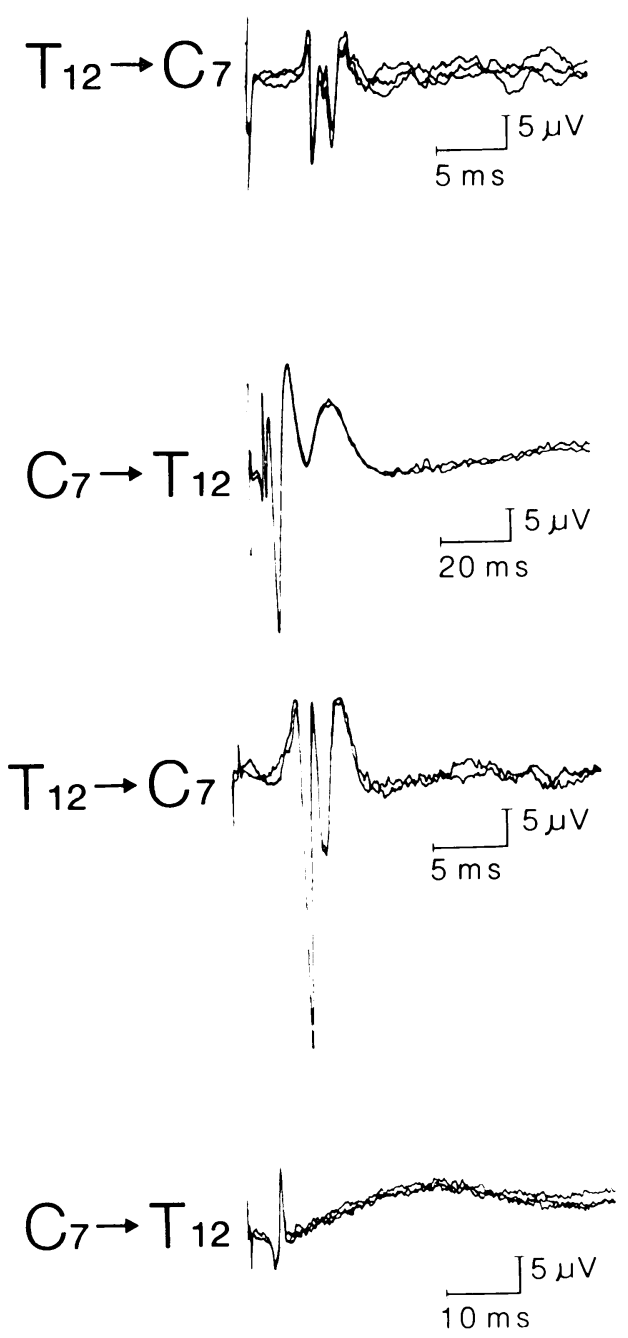

Fig. 2 Cord dorsum potentials recorded from the posterior epidural space in a normal subject (a) and in a patient suffering from amyotrophic lateral sclerosis $(b)$. UN $\rightarrow C 7, C D P$ recorded from the $C 7$ vertebral level, corresponding to the $C 8$ spinal segment, in response to ulnar nerve stimulation at the wrist. $P 1, N 1, P 2 F$, and $P 2 S$ denote the initially positive spike, the sharp negative wave, the first $(P 2 F)$ and second $(P 2 S)$ peaks of the slow positive wave, respectively. Note the prolonged negative wave which sometimes occurs (Shimoji et al., 1976) after the P2 wave in recording from a normal subject. $T N \rightarrow T 12, C D P$ recorded from the $T 12$ vertebral level, corresponding to the $S 1$ spinal segment, elicited by tibial nerve stimulation at the popliteal space. $T 12 \rightarrow C 7, C D P$ recorded from the $C 7$ vertebral level evoked by weak epidural stimulation at the $T 12$ vertebral level. $C 7 \rightarrow T 12, C D P$ recorded from the $T 12$ vertebral level by weak epidural stimulation at the $C 7$ vertebral level. Note the appearance of slow potentials, similar to the N1 and P2 waves evoked by peripheral nerve stimulation in the normal subject but not in the patient suffering from amyotrophic lateral sclerosis. Positivity is upward in all tracings. Each tracing is the average of 50 individual responses, obtained with an averaging computer (sampling rate of $10-50$ point $\mathrm{s} / \mathrm{ms}$ ), and plotted on an $X-Y$ recorder. The reference electrodes in recording from cervical and lumbar levels were placed on the skin surface of $C 7$ and $T 12$ spines, respectively. The time constant used was $0.3 \mathrm{~s}$ for all responses. 
Table Central peak latency of each component in human CDP and approximate conduction velocity through the cord. Central latency was measured from the initial positive peak of the spike. An approximate conduction velocity was calculated from the distance between stimulating and recording electrodes situated epidurally, and the onset time of the first spike. All values represent mean $+S E M$; values in parentheses indicate number of subjects

\begin{tabular}{|c|c|c|c|c|c|c|c|c|c|c|c|}
\hline \multicolumn{7}{|c|}{ Segmentally evoked CDP (ms) } & \multicolumn{3}{|c|}{ Suprasegmentally evoked CDP (ms) } & \multicolumn{2}{|c|}{ Conduction velocity $(\mathrm{m} / \mathrm{s})$} \\
\hline & $N 1$ & & $P 2 F$ & & $P 2 S$ & & $\begin{array}{l}\text { Sharp } \\
\text { negative wave }\end{array}$ & $\begin{array}{l}\text { First peak } \\
\text { of positive } \\
\text { wave }\end{array}$ & $\begin{array}{l}\text { Second } \\
\text { peak of } \\
\text { positive } \\
\text { wave }\end{array}$ & Ascending & Descending \\
\hline \multirow{3}{*}{$\begin{array}{l}\text { Normal } \\
\text { subjects }\end{array}$} & 3.50 & $\therefore 0.28$ & 7.32 & 0.31 & 21.30 & $=0.53$ & $2.55=0.32$ & $6.25 \cdot 0.22$ & $18.9 \because 0.6$ & $85.5 \div 6.6$ & $105.8: 3.8$ \\
\hline & (11) & & (11) & & (11) & & (11) & (11) & (11) & (6) & (6) \\
\hline & & & & & & & $* P<0.05$ & $\mathrm{P}<0.05$ & $\mathrm{P}<0.05$ & & \\
\hline $\begin{array}{l}\text { Amyotrophic } \\
\text { lateral }\end{array}$ & 3.31 & 0.27 & 6.92 & 0.38 & 22.08 & 0.54 & & & & $85.6: 9.8$ & $112.3: 9.8$ \\
\hline sclerosis & $(6)$ & & $(6)$ & & $(6)$ & & & & & $(5)$ & $(5)$ \\
\hline ratients & $\mathrm{NS}$ & & NS & & NS & & & & & NS & NS \\
\hline
\end{tabular}

* Values were compared by using Student's $t$ test between the similar components in segmentally and suprasegmentally evoked CDPs.

$\uparrow$ Values were also compared between normal subjects and patients with amyotrophic lateral szlerosis (NS $=$ not significant).

Schmidt, 1971; Wall, 1967). The finding of peak latency differences between the slow potentials evoked suprasegmentally and segmentally suggests that there are some different interneuronal pathways to produce PAD in human spinal cord. Absence of these slow potentials with preservation of the initial spike in the CDP elicited by descending volleys in patients with amyotrophic lateral sclerosis, in which the pyramidal tract is affected (Oppenheimer, 1976), may support this thesis. The spikes produced by descending volleys in the spinal cord of patients with amyotrophic lateral sclerosis, therefore, are presumably the antidromic impulses along the dorsal column. Thus, epidural recording of human CDPs evoked suprasegmentally may be useful in the study of neurological diseases which affect spinal cord function.

This work was supported in part by the Japanese Ministry of Education.

\section{References}

Andersen, P., Eccles, J. C., and Sears, T. A. (1964). Cortically evoked depolarization of primary afferent fibers in the spinal cord. Journal of Neurophysiology, 27, 63-77.

Cracco, R. Q. (1973). Spinal evoked response: peripheral nerve stimulation in man. Electroencephalography and Clinical Neurophysiology, 35, 379386.

Eccles, J. C. (1964). The Physiology of Synapses, pp. 220-238. Springer-Verlag: New York.

Ertekin, C. (1976). Studies on human evoked electrospinogram. 1. The origin of the segmental evoked potentials. Acta Neurologica Scandinavica, 53, 3-20. Fetz, E. E. (1968). Pyramidal tract effects on interneurons in the cat lumbar dorsal horn. Journal of Neurophysiology, 31, 69-80.

Gasser, H. S., and Graham, H. T. (1933). Potentials produced in the spinal cord by stimulation of dorsal roots. American Journal of Physiology, 103, 303320.

Jones, S. J. (1977). Short latency potentials recorded from the neck and scalp following median nerve stimulation in man. Electroencephalography and Clinical Neurophysiology, 43, 853-863.

Magladery, J. W., Porter, W. E., Park, A. M., and Teasdall, R. D. (1951). Electrophysiological studies of nerve and reflex activity in normal man. IV. The two neurone reflex and identification of certain action potentials from spinal roots and cord. Bulletin of the Johns Hopkins Hospital, 88, 499-519.

Matthews, W. B., Beauchamp, M., and Small, D. G. (1974). Cervical somatosensory evoked responses in man. Nature, 252, 230-232.

Oppenheimer, D. R. (1976). Diseases of the basal ganglia, cerebellum and motor neurons. In Greenfield's Neuropathology, third edition, pp. 608-651. Edited by W. Blackwood and J. A. N. Corsellis. Edward Arnold: London.

Schmidt, R. F. (1971). Presynaptic inhibition in the vertebrate central nervous system. Ergebnis der Physiologie, 63, 20-101.

Shimoji, K., Higashi, H., and Kano, T. (1971). Epidural recording of spinal electrogram in man. Electroencephalography and Clinical Neurophysiology, 30, 236-239.

Shimoji, K., Matsuki, M., Ito, Y., Masuko, K., Maruyama, M., Iwane, T., and Aida, S. (1976). Interactions of human cord dorsum potential. Journal of Applied Physiology, 40, 79-84. 
Shimoji, K., Matsuki, M., and Shimizu, H. (1977). Wave-form characteristics and spatial distribution of evoked spinal electrogram in man. Journal of Neurosurgery, 46, 304-313.

Shimoji, K., Shimizu, H., and Maruyama, Y. (1978). Origin of somato-sensory evoked responses recorded from the cervical skin surface. Journal of Neurosurgery, 48, 980-984.

Wall, P. D. (1967). The laminar organisation of dorsal horn and effects of descending impulses. Journal of Physiology, 188, 403-423. 